Trauma Berufskrankh 2008 · 10[Suppl 1]:99-106 DOI 10.1007/s10039-007-1310-8

Online publiziert: 2. Dezember 2007

c) Springer Medizin Verlag 2007

E.A.M. Neugebauer $\cdot$ T. Tecic

Institut für Forschung in der Operativen Medizin, Fakultät für Medizin,

Private Universität Witten/Herdecke, Campus Köln-Merheim, Köln

\title{
Lebensqualität nach Schwerstverletzung
}

ten, denen das traditionelle OutcomeKonzept nicht mehr genügt und die die Bedeutung der Berücksichtigung von hermeneutischen Zielvariablen (z. B. Lebensqualität, Patientenzufriedenheit, Befindlichkeit, Autonomie) deutlich machen:

- Bevölkerungsentwicklung,

- Fortschritte in der präklinischen und klinischen Versorgung,

- Wandel im Gesundheitswesen.

steht eine sinkende Mortalitätsrate gegenüber, die unter anderem durch Präventivmaßnahmen sowie Fortschritte in der medizinischen Notfall- und Akutversorgung bedingt ist. In Abhängigkeit von der Verletzungsschwere überleben inzwischen durchschnittlich mehr als $80 \%$ der schwerstverletzten Patienten [33]. Angesichts dieser hohen Überlebensrate verlagert sich der Fokus der Aufmerksamkeit zunehmend auf die Qualität des Überlebens. Eine systematische Erforschung und valide Erfassung des Konstrukts Lebensqualität als Outcome-Maß mit dem Ziel adäquater Förderung und Verbesserung erscheint demnach mehr als dringlich.

\section{Zum Begriff des Outcome}

Im internationalen medizinischen Sprachgebrauch wird das Ergebnis, das durch eine medizinische Therapie erzielt wird, als „outcome" bezeichnet. Durch die Definition verschiedener Ziele in Form von Zielgrößen oder Endpunkten wird das Ergebnis klassifiziert. Traditionelle Endpunkte in der Medizin sind die Überlebensrate, Komplikationsraten (z. B. Pneumonie, Rezidivraten) oder verschiedene Laborwerte. Meist sind Endpunkte in der physikalischen Realität vorhanden und beobachtbar.

In den vergangenen Jahrzehnten sind drei wichtige Entwicklungen zu beobach-
Im Rahmen der Bevölkerungsentwicklung mit einem steigenden Anteil älterer Menschen und einem Anstieg chronischer Erkrankungen, einhergehend mit dem Rückgang tödlicher Infektionen, ist die alleinige Frage nach dem Überleben nicht ausreichend. Die herkömmlichen Indikatoren des Gesundheitszustands, wie Symptomreduktion und Überleben, müssen um die Betrachtung der Qualität des Überlebens erweitert werden. Die Frage nach einer Verbesserung der Lebensqualität mit oder trotz chronischer Erkrankungen oder Beeinträchtigungen rückt zunehmend in den Vordergrund.

Weitere Entwicklungen, die zum Interesse an Lebensqualitätsforschung entscheidend beitragen, sind

- der medizinische Fortschritt und

- Maßnahmen der Unfallprävention.

Ständige Verbesserungen der medizinischen Versorgung in der Akut- und Intensivbehandlung führen zu einer erhöhten Überlebensrate. In diesem Zusammenhang ist auch die Optimierung der unfallpräventiven Maßnahmen zu nennen, wie z. B. Airbags in Fahrzeugen, die zu einer höheren Überlebenswahrscheinlichkeit nach Unfällen führen. Gestiegene Überlebensraten wiederum machen auch hier das Überdenken bzw. Erweitern der traditionellen Endpunkte in der Medizin notwendig und steigern die Bedeutung der Lebensqualität $[6,14,33,51,53]$. Für die Outcome-Forschung und medizinische Behandlung nach einem Unfall ergibt sich somit nicht nur die Frage, $o b$ ein Patient einen schweren Unfall überlebt, sondern von wachsender Bedeutung ist, wie er ihn überlebt [6]. Aus diesem Grund ist eine Erweiterung des Outcome-Konzepts erforderlich, die neben den objektiven "harten" medizinischen OutcomeWerten (Laborwerte, Funktion, Komplikationen), die subjektive Einschätzung des Patienten hinsichtlich seiner empfundenen Lebensqualität berücksichtigt. Weiterhin können Therapieformen - entsprechend dem Grad der Verbesserung, die sie in Bezug auf die Lebensqualität erreichen - bewertet werden. Die aus objektiven und subjektiven Aspekten integrierte Sichtweise der Outcome-Skalen ist eng mit Konzepten von Krankheit und Gesundheit verbunden. Ihre Konzeptualisierung muss im Konsens zwischen Patienten, Ärzten und Gesellschaft festgelegt werden. Dies bedeutet ein erhebliches Umdenken. Nicht mehr nur der Arzt legt die Zielkriterien seiner Intervention fest, sondern auch der Patient wird mit seiner subjektiven Meinung, seiner Einstellung und seinem sozialen und kulturellen Hintergrund hierbei einbezogen. Noch immer sind an diesem Punkt große Widerstände seitens der Ärzte zu überwinden: Ihr Glaube an objektive Parameter wie Mortalität, Komplikationsraten, Winkelmaße oder Laborwerte ist, wenn es um die Beurteilung des Krankheitsverlaufs oder Nutzens einer Intervention geht, oft sehr viel größer. Dies geht nach Lorenz [35] auf 
die Überbewertung von klinischen, biochemischen/molekularbiologischen oder physiologischen Indizes zurück, nach denen das gesamte Medizinstudium ausgelegt ist. Dies bedeutet nun aber nicht, dass die Bewertung z. B. der Mortalität und der Komplikationsraten keine Bedeutung mehr hat; ihr Wert ist aber limitierter und beschränkt sich auf Szenarien mit hohen Mortalitätsraten.

Aus Patientensicht müssen neue Outcome-Parameter hinzukommen, wie:

- funktioneller Status,

- emotionale Gesundheit,

- soziale Interaktion, kognitive Funktion,

- Grad der Zufriedenheit und

- andere Gesundheitsindikatoren.

Das Ziel der Intervention wird in Zukunft noch stärker im Konsens zwischen Arzt und Patient festgelegt („shared decision making").

\section{Langzeitfolgen nach Trauma}

Traumatisierte Patienten leiden häufig noch Jahre nach dem Unfall unter dessen Spätfolgen. Diese bestehen aus physischen Verletzungen, körperlichen Funktionseinschränkungen und Schmerzen. Hinzu kommen die auf den ersten Blick nicht ersichtlichen, jedoch stark die Lebensqualität beeinträchtigenden psychischen Beschwerden. Im Unfallszenario erlittene extreme Gefühle der Hilflosigkeit, des „Ausgeliefertseins“, der eigenen Verletzlichkeit und Verwundbarkeit können die psychisch regulativen Mechanismen überfordern und die Ausprägung von Angsterkrankungen, Depressionen sowie die Entwicklung von posttraumatischen Stresssyndromen begünstigen.

\section{Physische Verletzungen}

Analysen des Traumaregisters $(n=20.815)$ [33] der Deutschen Gesellschaft für Unfallchirurgie (DGU) zufolge sind die häufigsten Verletzungen nach einem Unfall

- Kopfverletzungen (58,0\%),

- Thoraxverletzungen $(56,1 \%)$,

- Extremitätenverletzungen (38,6\%) und

- Abdominalverletzungen (24,5\%).
Das Verletzungsmuster und der Verletzungsgrad hängen unter anderem von der Art und Schwere des Unfalls ab. Bei Unfällen mit Fußgängern und Fahrradfahrern treten vorrangig Schädelverletzungen auf, wohingegen bei Motorradfahrern vermehrt Frakturen der unteren Extremitäten vorkommen. Thorax- und Beckenläsionen sind die häufigsten Verletzungsregionen bei PKW-Unfällen [61]. Patienten mit Extremitätenverletzungen, die 56-93\% aller Verletzungen ausmachen $[4,47,54]$, müssen oft langwierige, Wochen bis Monate dauernde, nicht selten auch mit Komplikationen verbundene, Behandlungsverläufe über sich ergehen lassen.

Mehr als andere Verletzungen führen Extremitätenverletzungen zu Einschränkungen der Mobilität und dazu, dass die für den gesunden Menschen normalen Aktivitäten des alltäglichen Lebens - wie Gehen, Treppensteigen und Körperhygiene (Waschen, Toilettengänge etc.) - für den Unfallpatienten ein ernsthaftes Problem darstellen. Die Einschränkung der Beweglichkeit und Abhängigkeit von Hilfsmitteln wie Gehstützen bedeuten einen erheblichen Verlust an Autonomie und Lebensqualität und können die Entwicklung von psychischen Belastungszuständen, Depression und sozialer Deprivation forcieren. Outcome-Studien zeigen, dass Unfallpatienten noch 1-2 Jahre nach dem Unfall Probleme mit der Mobilität, der Selbstversorgung und dem Ausführen alltäglicher Aktivitäten haben [59, 63].

\section{Psychische Folgen}

Folgen eines Traumas auf psychischer Ebene sind sehr häufig: So leiden 38\%$60 \%$ der Traumapatienten an Fahrphobien $[8,9,23,24,32,44,39,40,41]$, bei 8$42 \%$ der Patienten liegt eine Angststörung vor $[9,12,32,37,44,59]$ und $8-45 \%$ berichten über eine depressive Symptomatik [3, 24, 25, 32, 36, 39, 59].

Epidemiologische Studien haben zeigen können, dass eine Vielzahl von Patienten in der Zeit nach einem Verkehrsunfall eine posttraumatische Belastungsstörung (PTBS; engl. „posttraumatic stress disorder", PTSD) oder Symptome einer akuten Belastungsreaktion (ASD) ent- wickeln. Frommberger [18] kam zu einer Prävalenzrate von $18 \%$ innerhalb von 6 Monaten nach einem Verkehrsunfall. Nach 6 Monaten waren es noch $11 \%$. Andere Studien fanden, dass $4-68 \%$ der Traumapatienten eine PTBS bzw. Symptome einer PTBS entwickeln [13, 19, 27, 31, 37, 56, 57]. Zu diesen Symptomen zählen vor allem sich wiederholt aufdrängende Erinnerungen an das belastende traumatische Ereignis. Diese Erinnerungen gehen oft mit großer Angst einher, weshalb die Betroffenen sowohl diese Gedanken als auch Situationen, die sie daran erinnern könnten, meiden. Auf körperlicher Ebene sind häufig Schlafstörungen, Konzentrationsstörungen, Schreckhaftigkeit, Ruhelosigkeit und eine erhöhte Reizbarkeit die Folge [6o].

Weitere Bereiche, die noch ein Jahr nach dem Unfall Probleme bereiten, sind somatoforme Beschwerden $[3,56]$ und kognitive Beeinträchtigungen [59].

Studien zeigen, dass psychische Folgen eines Unfalls relativ unabhängig von der objektiven Unfallschwere sind. Das Auftreten psychischer Symptome korreliert nicht mit dem Verletzungsgrad des Patienten. Vielmehr scheint die subjektive Wahrnehmung der Traumaschwere relevant zu sein $[42,56,57,62]$. Der beste Prädiktor für die Entwicklung einer PTBS oder anderer psychiatrischer Auffälligkeiten nach einem Unfall scheint die subjektiv wahrgenommene Lebensbedrohung zu sein $[16,23,39,45]$. Weitere Prädiktoren, die für die Entwicklung psychischer Symptome identifiziert sind, sind das bewusste Miterleben des Unfalls [39], die prämorbide Persönlichkeit $[13,26]$ sowie die Verfügbarkeit traumarelevanter Bewältigungsstrategien und sozialer Ressourcen $[13,57]$. Frauen zeigen nach einem Unfall einen größeren Vertrauensverlust hinsichtlich des Fahrens, mehr Ängste und ein höheres Risiko, an PTBS zu erkranken, als Männer [13, 29, 38].

\section{Soziale und finanzielle Folgen}

$\mathrm{Zu}$ den Spätfolgen eines Traumas gehören neben körperlichen und psychischen Folgen auch soziale und finanzielle Konsequenzen. Von den Traumaopfern leiden 60-70\% 6 Monate später an diversen psychosozialen Belastungen [16, 36, 39]; sie 
berichten von Ehe- und Partnerschaftsproblemen $[12,50]$ und erschwerter beruflicher Wiedereingliederung. Selbst 46 Jahre nach einem schweren Unfall sind noch durchschnittlich 30-40\% der Patienten ganz oder teilweise berufsunfähig $[1,2,7,58]$.

Die beschriebenen Langzeitfolgen lassen auf eine erhebliche Minderung der Lebensqualität Betroffener schließen. Um aber gesicherte und valide Aussagen treffen und adäquate Interventionen ableiten zu können, ist eine Operationalisierung des Konstrukts unumgänglich.

Wie ist das Konzept der Lebensqualität allgemein und speziell für polytraumatisierte Patienten zu fassen und zuverlässig zu messen?

\section{Lebensqualität: Begrifflichkeit, Konzeption, Operationalisierung}

Der Begriff „Lebensqualität“ ist ein Modewort. Er wird meist völlig undifferenziert von der Werbung für alle Branchen und in der Politik eingesetzt. Politiker nutzten ihn als politisches Konzept und Schlagwort für demokratische und sozialpolitische Ziele und zur Konzeptualisierung des Grundrechts jeden Bürgers, nach persönlichem Wohlergehen und Glückseligkeit streben zu können $[22,55]$.

Auch in der sozialwissenschaftlichen Literatur (Soziologie, Politologie, Anthropologie, Psychologie) spielte der Begriff schon in den 4oer-Jahren eine große Rolle und diente - auch interkulturell - der Erforschung der Lebensqualität in verschiedenen Ländern, wobei Lebensqualität hier als sozioökonomische Ressource und Gesundheitsversorgung eines Staates definiert wurde, später auch als Ausdruck für „Lebenszufriedenheit“ und „Wohlbefinden“.

In Abgrenzung zu diesen soziologisch orientierten Lebensqualitätskonzepten verwendete die Medizin Anfang der 8oerJahre den Begriff „gesundheitsbezogene Lebensqualität“. Mit der Frage des Arztes „Wie geht es Ihnen?“ sollte zunehmend wichtig werden, wie der Patient sich fühlt und nicht nur, was der Arzt denkt, wie der Patient sich gemäß den vorliegenden medizinisch-technischen Daten fühlen müsste. Als eigenständiger Terminus wurde das Konzept Lebensqualität von der Konsen-

Trauma Berufskrankh 2008 · 10[Suppl 1]:99-106

(c) Springer Medizin Verlag 2007

\section{E.A.M. Neugebauer · T. Tecic Lebensqualität nach Schwerstverletzung}

\section{Zusammenfassung}

Eine Vielzahl von Präventivmaßnahmen sowie Fortschritte in der medizinischen Akutversorgung von Unfallpatienten führten in den letzten Jahren zu deutlich gesunkenen Mortalitätsraten. Die Frage, ob ein Patient überlebt, weicht somit verstärkt der Frage, wie er überlebt. Der vorliegende Artikel setzt sich kritisch mit dem traditionellen OutcomeKonzept in der Medizin auseinander und plädiert für eine stärkere Einbeziehung von $\mathrm{Pa}$ tientenwerten hinsichtlich der Einschätzung ihrer persönlichen Behandlungsziele. Einem Überblick über die physischen und psychischen Langzeitfolgen nach einem Trauma folgt eine Erörterung des häufig missbrauchten Begriffs der "Lebensqualität". Auf der Grundlage einer allgemein gültigen Definition des Konstrukts Lebensqualität wurde ein Instrument zur Erfassung von Lebens-

\section{Quality of life after severe injuries}

\section{Abstract}

Preventive measures and rapid progress in the acute medical care of accident patients has, in recent years led to a clear reduction in the mortality rate. The question of whether patients will survive has been substantially replaced by the question how they will survive. The present article critically deals with traditional outcome measures used in medicine and pleads for a stronger involvement of patients'self-reported assessment related to impaired physical and mental conditions. After an overview of long-term physical and psychiatric consequences of trauma patients, the often misused term "quality of life" is discussed. On the basis of a commonly accepted definition of the concept of quality of life, an instrument was developed to assess the quality of life in multiply injured patients. By us- qualität entwickelt, welches vor allem durch das Modul TOP (Trauma Outcome Profile) den besonderen Umständen und Konsequenzen schwerstverletzter Patienten gerecht wird. Abschließend wird der praktische Nutzen der Erfassung subjektiv wahrgenommener Lebensqualität für Patienten, Kliniker und Gesellschaft dargestellt. Schließlich wird ein Ausblick gegeben, wie der mangelhaften Anwendung der Erfassung von Lebensqualität im Klinikalltag durch eine computergestützte, leicht handhabbare und automatisch auswertende Version des POLO-Charts begegnet werden kann.

\section{Schlüsselwörter}

Outcome · Polytrauma · Physische und psychische Langzeitfolgen - Lebensqualität . POLO-Chart ing a specific module TOP (Trauma Outcome Profile) the particular conditions and consequences of polytrauma patients are taken into account. Finally, the great benefit of measuring subjectively perceived quality of life is shown for patients' treatment as well as for physicians and society. A future prospect is given to enable the still insufficiently implemented quality of life measurement in clinical daily practice. For this purpose a computer-assisted version of the POLO-Chart will be generated, which is user-friendly and evaluation is carried out automatically.

\section{Keywords}

Outcome Polytrauma Physical and psychiatric consequences of trauma - Quality of life · POLO-Chart 


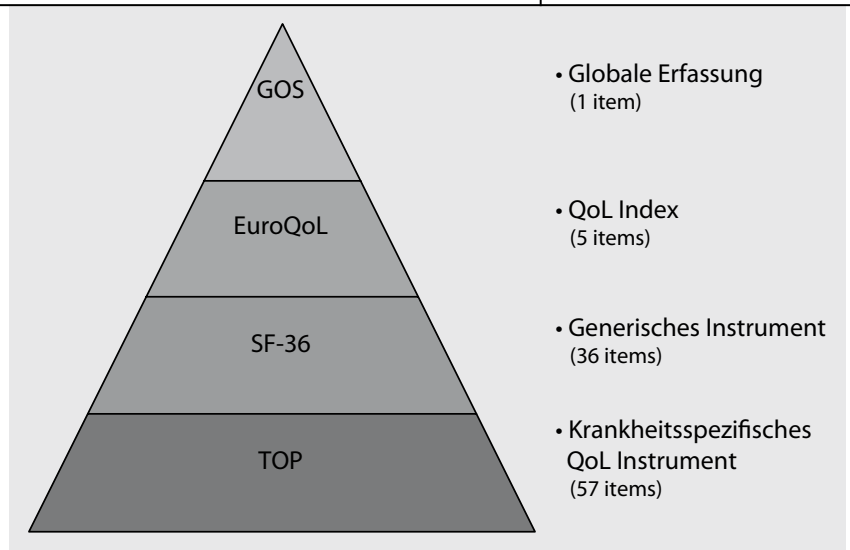

Abb. $1<$ Aufbau des Polytrauma-Outcome (POLO)-Charts

suskonferenz in Meran schließlich als „persönliche Wahrnehmung des eigenen körperlichen und psychischen Befindens und der sozialen Integration einer Person nach Einbeziehung von Krankheit und Gesundheit" definiert [48].

Nach diesem Konzept werden körperliche Beschwerden eines Patienten nicht nur anhand objektiver biomedizinischer Parameter (Mortalitätsrate, Komplikationsrate, Laborwerte) beurteilt; vielmehr bezieht man die subjektiven, patientenorientierten Aspekte - vom Patienten selbst berichtet - für die Evaluation des Gesamtzustands mit ein.

Großangelegte qualitative Studien zur patientenbezogenen Definition der Lebensqualität zeigen, dass die Dimensionen intersubjektiv vergleichbar sind. In einer offenen Befragung zum Begriff Lebensqualität mit 1000 Personen zeigte die Inhaltsanalyse der Antworten, dass es sich im Wesentlichen um Dimensionen der körperlichen, psychischen, sozialen und funktionalen Gesundheit handelt, welche die Personen als bedeutsam und indikativ für ihr Wohlbefinden angaben [10]. In einem groß angelegten interkulturellen Vergleich kam man zu den gleichen Ergebnissen. Lebensqualität konnte somit letztendlich im Rahmen der Meraner Konsensuskonferenz als multidimensionales Konstrukt mit folgenden Komponenten definiert werden:

- physisch (Mobilität, körperliche Funktionsfähigkeit, medizinische Parameter),

- symptombeschreibend (Schmerzen),

- psychisch (Emotionen, Kognitionen) und

- sozial (Familie, Arbeit) definiert werden konnte [48].
Im Rahmen dieser Konferenz (an der Chirurgen, Methodiker, Psychologen etc. teilnahmen) wurden Standards formuliert, um das multidimensionale Konstrukt zu operationalisieren und für verschiedene Patientengruppen konkret anwendbar zu machen. Diese Standards beinhalten allgemeine Richtlinien für die Wahl eines Messinstruments (wie z. B. die genaue Identifikation des Problems, die Zuordnung zu den definierten Lebensqualitätskomponenten und Prüfung des entsprechenden Instruments nach Anwendbarkeit und statistischer Güte). Vor dem Hintergrund geringer Korrelation zwischen selbstberichteten Erhebungen (Patientenaussagen) und den ärztlichen Einschätzungen [15] einigte man sich auf die Form der Befragungen. Sie sollten in eindeutig selbstberichtender Form („,selfreporting questionnaires") oder als Interviews durchgeführt werden, um die Subjektivität der Daten zu gewährleisten und die Eigenkontrolle des Patienten zu erhöhen [30].

In den letzten Jahren der Lebensqualitätsforschung ist die Operationalisierung des Konstrukts mit einer Reihe von Messinstrumenten zur Erfassung der gesundheitsbezogenen Lebensqualität weiter vorangetrieben worden. Diese Instrumente sollen die verschiedenen Komponenten (s. oben) erfassen. Sie lassen sich in globale Instrumente und in krankheitsspezifische Instrumente differenzieren. Mit der globalen, krankheitsübergreifenden Erfassungsform, soll ein Indikator für die subjektive Gesundheit von Populationen gewonnen werden, unabhängig vom aktuellen Gesundheitszustand.

Ein populäres und bewährtes Verfahren zur Erfassung der gesundheitsbezogenen Lebensqualität, das einerseits kul- turübergreifend für den internationalen Gebrauch verwendet werden kann, darüber hinaus aber auch bezüglich seiner psychometrischen Qualitäten, Ökonomie und Vielfalt der Einsatzmöglichkeiten international führend ist, stellt der 36-Item Short Form Health Survey (SF36) dar. Dieser Fragebogen hat eine fundierte 20-jährige Entwicklungsgeschichte und ist dabei eng mit der Lebensqualitätsforschung verknüpft.

In einem Vergleich verschiedener gesundheitsbezogener Lebensqualitätsinstrumente bei chronischen Schmerzpatienten wies der SF-36 die besten psychometrischen Eigenschaften auf; Schwächen ließen sich durch Einsatz von Zusatzmodulen kompensieren [21].

\section{Lebensqualität nach Polytrauma}

Das unfallbedingte Polytrauma ist durch die Erfahrung einer extremen Ausnahmesituation für Körper und Seele gekennzeichnet. Ein allgemeines Lebensqualitätskonzept, wie es dem SF-36 zu Grunde liegt, kann den spezifischen Umständen und Konsequenzen schwerverletzter, traumatisierter Patienten nicht genügen. Besonderheiten, die sich aufgrund des Ereignisses ergeben, werden unzureichend beziehungsweise gar nicht beachtet. Das Unfallgeschehen wird von den Betroffenen als unvorhersehbar, plötzlich, gewaltig und lebensbedrohlich erlebt. Aus psychologischer Perspektive wird ihre Weltsicht massiv erschüttert, gingen sie doch jahrelang davon aus, in einer sicheren, wohlmeinenden und bis zu einem gewissen Grad vorhersagbaren Umgebung zu leben. Zudem tritt eine enorme Verunsicherung hinsichtlich des eigenen Selbstbilds von Stärke und Kontrolle ein. Das Patientenkollektiv polytraumatisierter Menschen stellt somit auch unter psychologischen Gesichtspunkten eine sehr spezifische und stark beanspruchte Gruppe dar, die sich neben schweren, andauernden, körperlichen Beschwerden und Schmerzen auch großen und gravierenden Umorientierungen im privaten und beruflichen Bereich gegenüber sieht.

Mit der Absicht, ein standardisiertes und auf die besondere Situation von Unfallpatienten ausgerichtetes Messinstrument zur Lebensqualität zu etablieren, 


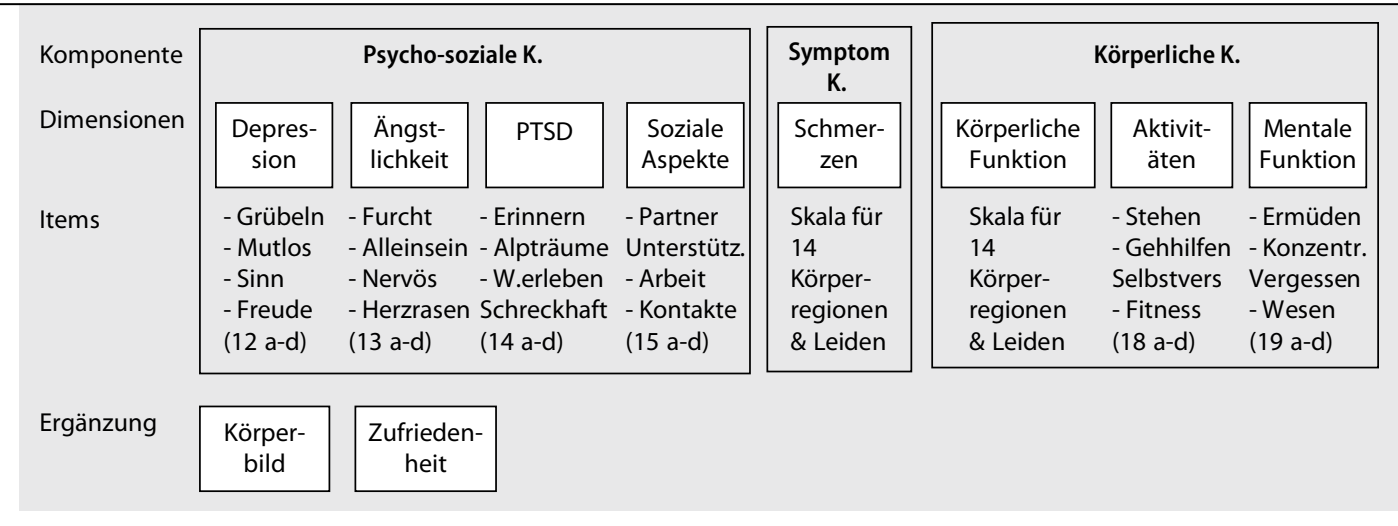

Abb. $2 \triangleright$ Struktur des Trauma Outcome Profile (TOP) als Teil des POLO-Charts

wurde in der integrierten Arbeitsgruppe Neugebauer/Bouillon und der AG Polytrauma der DGU der Polytrauma-Outcome-Chart (POLO-Chart) entwickelt, welcher sich durch seinen modularen Aufbau auszeichnet ( $\bullet$ Abb. 1).

Dieses Instrument beinhaltet die folgenden drei Messinstrumente die nach den Empfehlungen der internationalen Lebensqualitätskonferenz (1999) in allen Lebensqualitätsstudien unabhängig vom Patientenkollektiv angewendet werden [49]:

- Glasgow Outcome Scale (GOS)

- European Quality of Life Index (EuroQoL) und

- SF-36.

Der POLO-Chart enthält zusätzlich das weltweit erste traumaspezifische Lebensqualitätsmodul, das

\section{- Trauma Outcome Profile (TOP).}

Alle vier relevanten Komponenten der gesundheitsbezogenen Lebensqualität (physisch, psychisch, sozial und symptombeschreibend) werden auf 10 Dimensionen des TOP erfasst. Dabei wird die psychosoziale Komponente durch dien Dimensionen Depression, Ängstlichkeit, posttraumatischer Stress und soziale Aspekte dargestellt, die Symptomkomponente wird durch die Dimension Schmerzen repräsentiert. Die körperliche Komponente setzt sich aus den Dimensionen körperliche Funktion, Aktivitäten und mentale Funktion zusammen. Zusätzlich werden zwei einzelne Fragen zum Körperbild und zur allgemeinen Zufriedenheit erfasst. - Abb. 2 stellt die Struktur des TOP graphisch dar.

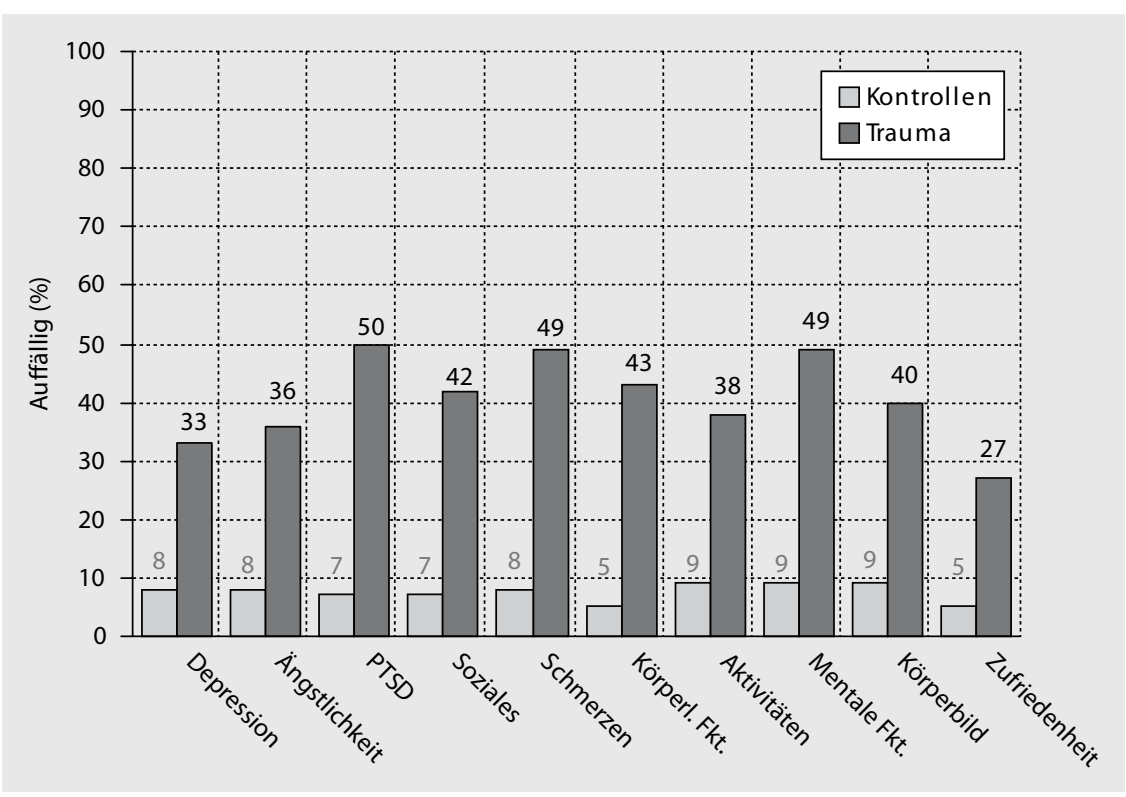

Abb. $3 \Delta$ Anteil auffälliger Personen in Trauma- und Kontrollgruppe auf den 10 Dimensionen des TOP (nach [34])

In einer Validierungsstudie [34], die in der Zeit von Juli 2001 bis August 2003 in 8 teilnehmenden deutschen und österreichischen Kliniken an insgesamt 338 Teilnehmern (172 Traumapatienten, $166 \mathrm{~Pa}$ tienten der Kontrollgruppe) durchgeführt wurde, erwies sich das Modul TOP über alle Dimensionen hinweg als ein geeignetes Maß für die Beurteilung der Lebensqualität speziell von Unfallpatienten. Traumatisierte Patienten zeigten im Vergleich zur Kontrollgruppe signifikant schlechtere Werte.

Auch zwei Jahre nach einem Unfall sind große Diskrepanzen hinsichtlich erfahrener Lebensqualität zwischen Traumapatienten und Kontrollprobanden zu verzeichnen [34]. Der Anteil auffälliger Personen in der Gruppe traumatisierter Patienten übersteigt in allen 10 Skalen des TOP deutlich die Auftretenshäu- figkeit Auffälliger bei nichttraumatisierten Probanden.

\section{Schmerzen und Lebensqualität}

Das meistgenannte Symptom nach einem Unfall ist der Schmerz. Gehling et al. [20] fanden, dass $61 \%$ der operierten Patienten 7-9 Monate nach dem Unfall an Schmerzen leiden. 3 Jahre nach dem Trauma klagen noch 21\% der Patienten über leichte bis schwere Schmerzen, unabhängig von der ermittelten Verletzungsschwere [43, 58]. 25\% der Patienten geben Schmerz als ihr größtes Problem an $[5,52]$ und erleben erhebliche Einschränkungen durch Schmerzen [28].

Die Schmerzskala des TOP ist sehr differenziert aufgebaut und erfasst die Schmerzintensität in 14 verschiedenen Körperregionen auf einer numerischen 

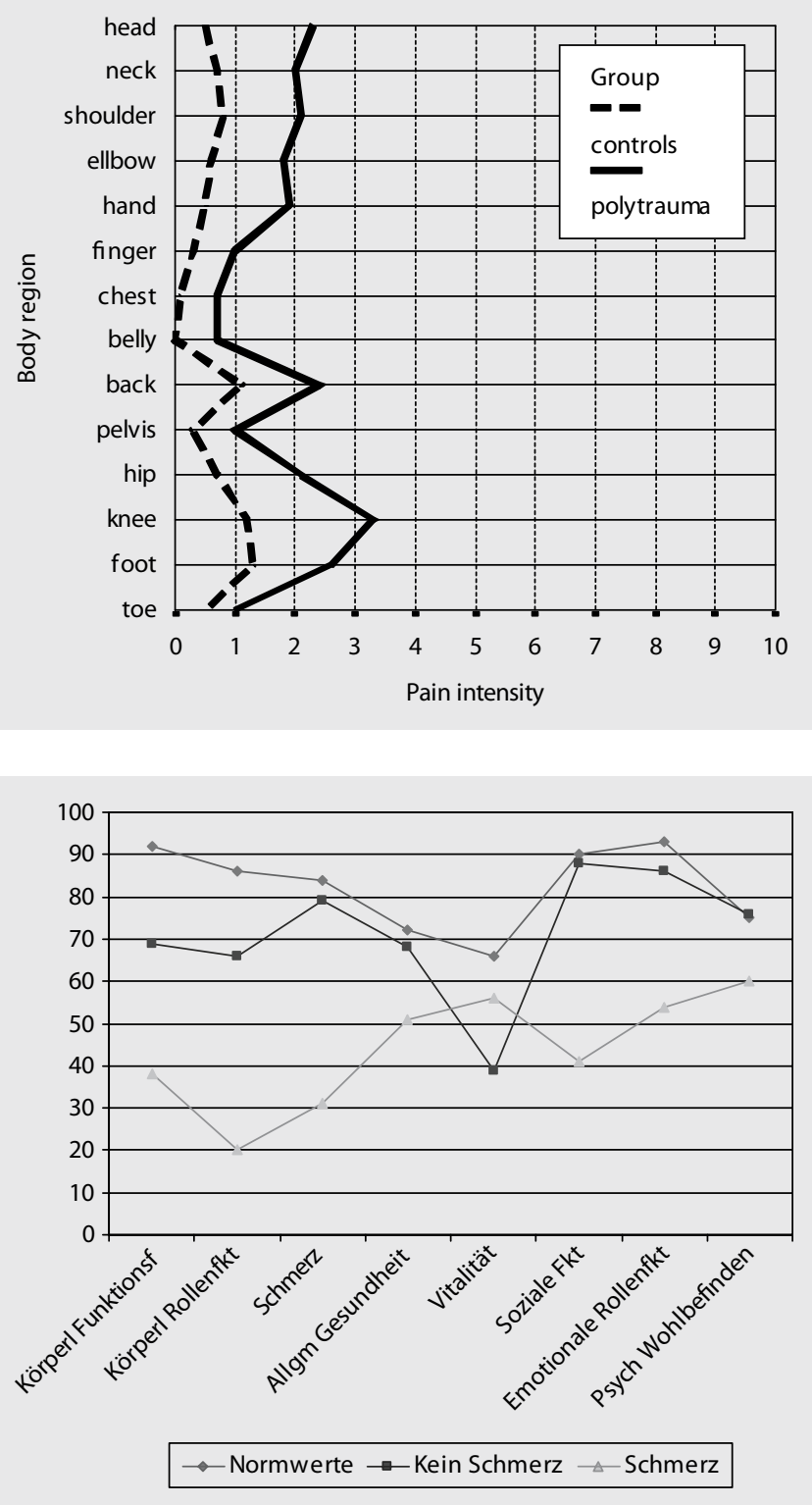

Abb. $4<$ Vergleich der Schmerzintensität von Polytraumapatienten 2 Jahre nach dem Unfall mit Kontrollprobanden

Abb. $5<$ Der Einfluss von Schmerzen auf die Lebensqualität polytraumatisierter Patienten (Erfassung mittels SF-36)

Ratingskala von o „keine Schmerzen“ bis 10 , unerträgliche Schmerzen“. Der Unterschied zwischen 44 Kontroll- und 59 Polytraumapatienten in der Schmerzwahrnehmung zwei Jahre nach Trauma ist in - Abb. 4 veranschaulicht. Der Abbildung sind die errechneten Mittelwerte der jeweiligen Gruppen für eine bestimmte Körperregion zu entnehmen. Demnach berichten Traumapatienten 2 Jahre nach einem Unfall, an stärkeren Schmerzen in allen Körperregionen zu leiden als die Kontrollgruppe.

Inwieweit sich Schmerzen auf die Lebensqualität auswirken, lässt sich aus - Abb. 5 ablesen, deren Daten an einer Population von 92 Traumapatienten gewonnen wurden [52]. Als Lebensqualitätsmaß wurde der SF-36 ein Jahr nach dem
Trauma eingesetzt. Zur Verdeutlichung des Einflusses von Schmerz auf die Lebensqualität wurde die Gesamtstichprobe in zwei Gruppen aufgeteilt: Polytraumapatienten mit Schmerzen wurden einer Gruppe von Patienten ohne Schmerzen gegenübergestellt.

Mit Ausnahme der Skala Vitalität schneidet die „Schmerzgruppe“ wesentlich schlechter ab als die Gruppe ohne Schmerzen. Das Resultat legt den Schluss nahe, die Komponente „Schmerz“ als stark moderierende Variable für die Lebensqualität anzunehmen.

Schmerzbewältigungstechniken und Schmerzmanagementstrategien gewinnen dadurch als Therapiemethoden vermehrt an Relevanz, um das Ziel einer Verbesserung der Lebensqualität zu erreichen.

\section{Nutzen der Erfassung von Lebensqualitätsdaten}

Welcher Nutzen lässt sich außerdem aus einer validen und reliablen Erfassung von Lebensqualitätsdaten bei Schwerstverletzten ziehen? Welche Erkenntnisse und Vorteile sind mit ihrer Hilfe, über eine bloße Dokumentation hinaus, für den $\mathrm{Pa}$ tienten, den Kliniker, die Forschung und die Gesellschaft zu ziehen?

Mit der Einbeziehung subjektiv empfundener Lebensqualität bei der Erfassung des Gesundheitszustands schwerstverletzter Patienten wird ein bislang noch unzureichend vollzogener, jedoch zwingend nötiger Paradigmenwechsel aufgezeigt: Lag es vormals im Ermessen des Arztes, den gesundheitlichen Zustand (Outcome) des Patienten anhand objektiver Parameter zu beurteilen, tritt nun zunehmend die subjektiv empfundene Wahrnehmung über das eigene Befinden des Patienten in den Vordergrund. Die Notwendigkeit, die Patientenwahrnehmung zu berücksichtigen, wird durch deutliche Hinweise darauf bestärkt, dass die Lebensqualität schwerstverletzter Patienten stärker von der subjektiven als von der objektiven Verletzungsschwere abhängt [51] .

Wer sonst, als der Patient selbst, vermag zu beurteilen, wie es um sein Wohlbefinden steht?

Die möglichst erschöpfende Erfassung der wahrgenommenen Lebensqualität in allen relevanten Bereichen (körperlich, psychisch, sozial) unter besonderer Berücksichtigung der spezifischen Umstände und Folgen polytraumatisierter Patienten erlaubt eine adäquate, auf die Bedürfnisse der Patienten ausgerichtete Versorgung und Behandlung. Die diagnostische Erfassung frühzeitiger psychischer und sozialer Folgen gehört bisher nicht zum Routineprogramm der Versorgung von Unfallopfern. Die in der Akutversorgung also meist vernachlässigten psychosozialen Probleme werden beim Lebensqualitätsansatz ausdrücklich mitberücksichtigt, sodass ein umfassenderes und differenzierteres Bild der Schwierigkeiten und Belastungen entsteht. Eine rechtzeitige Problemidentifizierung, die über die offensichtlichen körperlichen Beschwerden hinausgeht, gewährleistet die entsprechende fachspezifische Behandlung. So 
konnten in einer eigenen Interventionsstudie hinsichtlich der psychischen Belastungen nach Unfall bereits positive Effekte einer frühzeitigen psychotherapeutischen Intervention aufgezeigt werden [52].

Unerkannte und damit unbehandelte psychische Verletzungen können zu Chronifizierungen führen, wodurch der gesamte Heilungsprozess erheblich erschwert und verlängert sowie auch die Dauer der beruflichen Reintegration maßgeblich mitbestimmt wird. Studien [11, 46] konnten nachweisen, dass Unfallpatienten, die hinsichtlich Angst und Depressionen Auffälligkeiten zeigten, bedeutend mehr Zeit benötigten, wieder in ihren produktiven Lebensstil zurückzufinden. Der berufliche Ausfall bedeutet sowohl für den Einzelnen als auch für die Gesellschaft hohe Kosten und Defizite, die es durch die geeignete Diagnostik und anschließende angemessene, fachkundige Behandlung aufzufangen gilt.

Die Forschung zur Lebensqualität wird vorwiegend in ihrer Funktion als systematische Informationserfassungsquelle eines großen Patientenpools und zur Messung von Effektivität in Interventionsstudien wahrgenommen. Dieser umfangreichen Datenerhebung ist es zu verdanken, dass sich Kenntnisse über die psychosozialen Belastungen verbessern, die in der Vergangenheit meist unbedacht blieben. $\mathrm{Zu}$ dem lassen sich durch statistische Berechnungen mögliche Prädiktoren identifizieren, die das Ausmaß der Lebensqualität vorhersagen, wodurch sich bereits präventiv Maßnahmen zur Erhaltung von Lebensqualität ableiten ließen. Die konkrete Anwendung von Lebensqualitätsdaten zur Entscheidungsfindung beim einzelnen $\mathrm{Pa}$ tienten wurde jedoch lange Zeit vernachlässigt. Bislang erfuhr z. B. nur ein geringer Teil der Patienten die notwendige psychotherapeutische Versorgung [17].

Für den Unfallchirurgen, der aufgrund erschwerter Arbeitszeit- und Kontextbedingungen oftmals nicht die Zeit für ein problemorientiertes, umfangreiches Gespräch mit seinem Patienten aufbringen kann, birgt dessen Selbstauskunft in Form des Lebensqualitätsfragebogens deshalb wichtige Anhaltspunkte. Es werden Problembereiche aufgezeigt, die das Kompetenzfeld des Chirurgen überschreiten und deren Behandlung ihn überfordern wür- den. Eine notwendige psychotherapeutische Versorgung kann jedoch erkannt werden, sodass eine Delegation an kompetente Fachgruppen ermöglicht und der behandelnde Arzt gleichzeitig entlastet wird.

Ziel subjektiv erhobener Daten ist es, eine angemessene und vor allen Dingen individualisierte Therapie zu planen und $\mathrm{zu}$ initiieren. Die Vielfalt des menschlichen Daseins, die durch unterschiedliche lebensgeschichtliche Erfahrungen bedingt ist, lässt Lebensschwerpunkte heranreifen, die in der Bedeutung interindividuell erheblich variieren können. Gleiche körperliche Verletzungen mögen sich unterschiedlich stark auf die subjektiv empfundene Lebensqualität ausprägen. Die aktive Einbeziehung und Beurteilung der Situation durch den Patienten selbst ist ein großer Schritt in Richtung auf eine adäquate und somit förderliche Behandlung.

Zusammenfassend lässt sich feststellen, dass

- die Bedeutung von Lebensqualität in der Beurteilung der Behandlung er-

kannt wurde und

- es mit dem POLO-Chart heute ein valides Instrument zur Erfassung von Lebensqualität nach Trauma gibt.

Bezüglich des Konstrukts der Lebensqualität besteht jedoch noch ein Missverhältnis zwischen dem wachsenden Interesse innerhalb der Forschung und der Implementierung dieses Outcomes in der Praxis.

\section{Fazit}

Während die methodische Auseinandersetzung über die Erfassung der Lebensqualität und der theoretische Diskurs über das Konstrukt weit vorangeschritten sind, befinden sich die klinische Implementierung und der Nutzen der Lebensqualitätserhebung für den einzelnen Patienten noch in den Anfängen. In der unfallchirurgischen Behandlung und Therapieplanung ist der Nutzen von Lebensqualitätsdaten noch kaum bekannt. Gerade Unfallopfer leiden jedoch an Beeinträchtigungen in vielen Lebensbereichen, sodass eine frühe integrative Behandlung ihre Lebensqualität erheblich verbessern könnte.
Die Bedeutung und Erfassung der wahrgenommenen Lebensqualität durch valide Messinstrumente (POLO-Chart) sollte in das diagnostische Routineprogramm eines Klinikers eingebunden werden, um individuell optimierte und integrative Therapie- und Rehabilitationsmaßnahmen besser planen und durchführen zu können.

Um eine Verbreitung in den Klinikalltag zu fördern, wird in naher Zukunft angestrebt, den POLO-Chart als standardisierte, computergestütze Version in die Praxis zu überführen. Mit dem Ziel einer patientengerechten, leichten Anwendbarkeit und zeitnahen, automatisierten Auswertung soll die diagnostische Erfassung der subjektiv empfundenen Lebensqualität bei jedem Patienten eingesetzt werden. Unnötige und langwierige Strapazen sowie hohe Kosten durch Fehlbehandlungen können so zum Wohl aller Beteiligten vermieden werden.

\section{Korrespondenzadressen}

\section{Prof. Prof. h. c. Dr. E.A.M. Neugebauer}

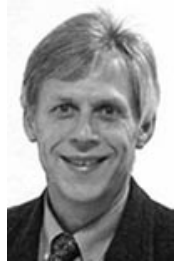

Institut für Forschung in der Operativen Medizin, Fakultät für Medizin, Private Universität Witten/Herdecke, Campus Köln-Merheim, Ostmerheimer Straße 200, 51109 Köln ifom-neugebauer-sek@ uni-wh.de

Interessenkonflikt. Der korrespondierende Autor gibt an, dass kein Interessenkonflikt besteht.

\section{Literatur}

1. Airey CM, Chell SM, Rigby AS et al. (2001) The epidemiology of disability and occupation handicap resulting from major traumatic injury. Disabil Rehabil 23: 509-515

2. Berger E (2005) Langzeitoutcome schwerverletzter Patienten: eine prospektive Studie zur Lebensqualität und beruflichen Reintegration bis 6 Jahre nach Unfalltrauma [Dissertation zur Erlangung der Doctorwürde rerum medicinalium]. Universität zu Köln, Köln

3. Blanchard EB, Hickling EJ, Taylor AE, Loos W (1995) Psychiatric morbidity associated with motor vehicle accidents. J Nerv Ment Dis 183: 495-504

4. Boddeker W, Reith HB, Smektala R et al. (1993) Analyse der polytraumatisierten Patienten von 1981 bis 1991. In: Kozuscheck W, Reith HB (Hrsg) Das Polytrauma - Diagnostik, Therapie. Krager, Freiburg 
5. Bostanci S (2005) Frühe somatische und psychosoziale Prädiktoren der gesundheitsbezogenen Lebensqualität bei schwerverletzten Patienten: Eine prospektive Studie. [Dissertation zur Erlangung des Doctorgrades rerum medicinalium]. Universität zu Köln, Köln

6. Bouillon B, Neugebauer E (1998) Outcome after polytrauma. Langenbecks Arch Surg 383: 228-234

7. Braithwaite IJ, Boot DA, Patterson M, Robinson A (1998) Disability after severe injury: five year follow up of a large cohort. Injury 29: 55-59

8. Bryant RA, Harvey AG (1990) Initial posttraumatic stress responses following motor vehicle accidents. J Trauma Stress 9: 223-235

9. Bryant RA, Harvey AG (1995) Psychological impairment following motor vehicle accidents. Aust J Public Health 19: 185-188

10. Bullinger M (1997) Gesundheitsbezogene Lebensqualität und subjektive Gesundheit. Psychother Psychosom Med Psychol 47: 76-91

11. Cornes P (1992) Return to work of road accident victims claiming compensation for personal injury. Injury 23: 256-260

12. Dittmer H, Bauer F (1987) Ergebnisse der psychischen, sozialen und somatischen Rehabilitation nach Polytrauma unter besonderer Berücksichtigung der Motorradfahrer. Z Unfallchir Versicherungsmed Berufskrankh 80: 84-88

13. Ehlers A, Mayou RA, Bryant B (1998) Psychological predictors of chronic posttraumatic stress disorder after motor vehicle accidents. J Abnorm Psychol 107: 508-519

14. Erli HJ, Fernandez V, Kugler J et al. (2000) Determinanten der globalen Lebensqualität nach Polytrauma. Chirurg 71: 1132-1137

15. Eypasch E, Troidl H, Wood-Dauphinee S et al. (1990) Quality of life and gastrointestinal surgery - a clinimetric approach to developing an instrument for its measurement. Theor Surg 5: 3-10

16. Feinstein A, Dolan R (1991) Predictors of post-traumatic stress disorder following physical trauma: an examination of the stressor criterion. Psychol Med 21: 85-91

17. Flatten G, Erli HJ, Hardorfer V et al. (2002) Psychotraumatologie in der Unfallmedizin. Zum Stand der psychischen Versorgung von Unfallopfern in Deutschland. Unfallchirurg 105: 231-236

18. Frommberger UH, Stieglitz RD, Nyberg E et al. (1998) Prediction of posttraumatic stress disorder by immediate reactions to trauma: a prospective study in road traffic accident victims. Eur Arch Psychiatry Clin Neurosci 248: 316-321

19. Fuglsang AK, Moergeli H, Schnyder U (2004) Does acute stress disorder predict post-traumatic stress disorder in traffic accident victims? Analysis of a self-report inventory. Nord J Psychiatry 58: 223229

20. Gehling M, Scheidt CE, Niebergall H et al. (1999) Persistent pain after elective trauma surgery. Acute Pain 2: 110-114

21. Gerbershagen HU, Lindena G, Korb J, Kramer S (2002) Gesundheitsbezogene Lebensqualität bei Patienten mit chronischen Schmerzen. Schmerz 16: 271-284

22. Glatzer W, ZapfW (Hrsg) (1984) Lebensqualität in der Bundesrepublik Deutschland. Campus, Frankfurt

23. Green MM, McFarlane AC, Hunter CE, Griggs WM (1993) Undiagnosed post-traumatic stress disorder following motor vehicle accidents. Med J Aust 159: 529-534

24. Hickling E, Blanchard E (1992) Post-traumatic stress disorder and motor vehicle accidents. J Anxiety Disord 6: 285-291
25. Hickling EJ, Blanchard EB (1997) The private practice psychologist and manual-based treatments: post-traumatic stress disorder secondary to motor vehicle accidents. Behav Res Ther 35: 191-203

26. Holeva V, Tarrier N, Wells A (2001) Prevalence and predictors of acute stress disorder and PTSD following road traffic accidents: Thought control strategies and social support. Behav Ther 32: 65-83

27. Holbrook TL, Hoyt DB, Coimbra R et al. (2005) High rates of acute stress disorder impact quality-of-life outcomes in injured adolescents: mechanism and gender predict acute stress disorder risk. J Trauma 59: $1126-1130$

28. Jones IH, Riley WT (1987) The post-accident syndrome: variations in the clinical picture. Aust N Z J Psychiatry 21: 560-567

29. Kessler RC, Sonnega A, Bromet E et al. (1995) Posttraumatic stress disorder in the national comorbidity survey. Arch Gen Psychiatry 52: 1048-1060

30. Koller M, Lorenz W (2002) Surgical decision making and behavior. Social psychology explanations and research perspectives. Chirurg 73: 846-854

31. Koren D, Arnon I, Klein E (1999) Acute stress response and posttraumatic stress disorder in traffic accident victims: a one-year prospective, followup study. Am J Psychiatry 156: 367-373

32. Kuch K, Cox BJ, Evans R, Shulman I (1994) Phobias, panic and pain in 55 survivors of road vehicle accidents. J Anxiety Disord 8: 181-187

33. Lefering R (2005) Jahresbericht des Traumaregisters der Arbeitsgemeinschaft "Polytrauma“ der Deutschen Gesellschaft für Unfallchirurgie (DGU) für $\mathrm{AG}$ „Polytrauma“ der DGU. Arbeitsgemeinschaft „Polytrauma“ der Deutschen Gesellschaft für Unfallchriurgie (DGU)

34. Lefering R, Ottlik Y, Pirente N et al. (2006) Quality of Life after multiple trauma: validation and population norm of the Polytrauma Outcome (POLO)Chart. In Vorbereitung

35. Lorenz W (1998) Outcome: definition and methods of evaluation. In: Troidl H, McKneally MF, Mulder DS et al. (eds) Surgical Research: Basic principles and clinical practice. Springer, Berlin Heidelberg New York, pp 513-520

36. Malt U (1988) The long-term psychiatric consequences of accidental injury. Br J Psychiatry 153: 810-818

37. Mason S, Wardrope J, Turpin G, Rowlands A (2002) The psychological burden of injury: an 18 month prospective cohort study. Emerg Med J 19: 400404

38. Mayou R, Simkin S, Threlfall J (1991) The effects of road traffic accidents on driving behaviour. Injury 22: 365-368

39. Mayou R, Bryant B, Duthie R (1993) Psychiatric consequences of road traffic accidents. BMJ 307 : 647-651

40. Mayou R, Tyndel S, Bryant B (1997) Long-term outcome of motor vehicle accident injury. Psychosom Med 59: 578-584

41. Mayou RA, Black J, Bryant B (2000) Unconsciousness, amnesia and psychiatric symptoms following road traffic accident injury. Br J Psychiatry 177: 540-545

42. Mayou R, Bryant B (2001) Outcome in consecutive emergency department attenders following a road traffic accident. Br J Psychiatry 179: 528-534

43. Mayou RA, Ehlers A, Bryant B (2002) Posttraumatic stress disorder after motor vehicle accidents: 3 year follow-up of a prospective longitudinal study. Behav Res Ther 40: 665-675

44. Mayou R, Bryant B (2003) Consequences of road traffic accidents for different types of road user. Injury $34: 197-202$
45. Meyer C, Steil R (1998) Die posttraumatische Belastungsstörung nach Verkehrsunfällen. Unfallchirurg 101: 878-893

46. Michaels AJ, Michaels CE, Smith JS et al. (2000) Outcome from injury: general health, work status, and satisfaction 12 months after trauma. J Trauma 48: 841-848

47. Nast-Kolb D, Jochum $M$, Waydhas $C$, Schweiberer $L$ (1991) Die klinische Wertigkeit biochemischer Faktoren beim Polytrauma. Springer, Berlin

48. Neugebauer E, Troidl H, Wood-Dauphinee S et al. (1991) Quality of life assessment in surgery: Results of the Meran consensus conference development conference. Theor Surg 6: 123-137

49. Neugebauer E, Bouillon B, Bullinger M, Wood-Dauphinee $S$ (2002) Quality of life after multiple trauma-summary and recommendations of the consensus conference. Restor Neurol Neurosci 20: 161-167

50. Paar O, Kasperk R (1992) Langzeitverlauf nach PoIytrauma. Unfallchirurg 95: 78-82

51. Pirente N, Gregor A, Bouillon B, Neugebauer E (2001) Lebensqualität schwerstverletzter Patienten ein Jahr nach Trauma - eine matched pair Studie im Vergleich zu einer gesunden Kontrollgruppe. Unfallchirurg 104: 57-63

52. Pirente N (2005) Effekt frühzeitiger psychotherapeutischer Interventionen bei schwerverletzten Patienten auf Parameter der Lebensqualität und der beruflichen Rehabilitation. [Dissertation zur Erlangung des Doctorgrades rerum medicinalium]. Universität zu Köln, Köln

53. Regel G, Seekamp A, Takacs J et al. (1993) Rehabilitation und Reintegration polytraumatisierter Patienten. Unfallchirurg 96: 341-349

54. Siegel JH, Dunham CM (1987) Trauma, the disease of the 20th centrury. In: Siegel JH (ed) Trauma. Churchill Livingstone, New York

55. Spilker B (1996) Introduction to the field of quality of life trials. In: Spilker B (ed) Quality of life and Pharmaeconomics in clinical trials. Lippincott-Raven, Philadelphia, pp 1-10

56. Schnyder U, Moergeli HP, Nigg C et al. (2000) Early psychological reactions to life-threatening injuries. Crit Care Med 28: 86-92

57. Schnyder U, Moergeli H, Trentz O et al. (2001) Prediction of psychiatric morbidity in severely injured accident victims at one-year follow-up. Am J Respir Crit Care Med 164: 653-656

58. Sluis CK van der, Eisma WH, Groothoff JW, Duis HJ ten (1998) Long-term physical, psychological and social consequences of severe injuries. Injury 29: 281-285

59. Vles WJ, Steyerberg EW, Essink-Bot ML et al. (2005) Prevalence and determinants of disabilities and return to work after major trauma. J Trauma 58: 126135

60. Whiteneck G, Brooks CA, Mellick D et al. (2004) Population-based estimates of outcomes after hospitalization for traumatic brain injury in Colorado. Arch Phys Med Rehabil 85: S73-S81

61. Wick M, Ekkernkamp A, Muhr G (1997) [The epidemiology of multiple trauma]. Chirurg 68: 10531058

62. Zatzick DF, Kang S-M, Müller H-G et al. (2002) Predicting posttraumatic distress in hospitalized trauma survivors with acute injuries. Am J Psychiatry 159: 941-946

63. Zettl RP, Ruchholtz S, Lewan U et al. (2004) Lebensqualität polytraumatisierter Patienten 2 Jahre nach Unfall. Notfall Rettungsmed 7: 547-553 